\title{
Termination Stereochemistry in Asymmetric Anionic Polymerization of Triphenylmethyl Methacrylate. Methylation and Protonation of Oligomer Anions
}

\author{
Tamaki Nakano, Yoshio Okamoto, and Koichi Hatada* \\ Department of Applied Chemistry, School of Engineering, Nagoya University, \\ Chikusa-ku, Nagoya 464-01, Japan \\ * Department of Synthetic Chemistry, Faculty of Engineering Science, Osaka University, \\ Toyonaka, Osaka 560, Japan
}

(Received January 17, 1995)

\begin{abstract}
Oligomerization of triphenylmethyl methacrylate (TrMA) was carried out with the complex of 9-fluorenyllithium and $(-)$-sparteine in toluene at $-78^{\circ} \mathrm{C}$. The reaction was terminated by methylation using methyl iodide and methyl iodide- $d_{3}$ or by protonation using methanol, butanol, 2-propanol, and $t$-butanol. The resulting oligomers were converted to oligo(methyl methacrylate) [oligo(MMA)]; the oligomers were first separated in terms of degree of polymerization and then into diastereomers. The individual diastereomers were resolved into enantiomers and the ratio of isomers was determined for each oligomer. Methylation with methyl iodide- $d_{3}$ of dimer anion occurred stereoselectively. Protonation stereochemistry was strongly affected by stereostructure of the protonating alcohols. The effect of alcohol was more obvious for the anions which were less active toward TrMA monomer.
\end{abstract}

KEY WORDS Triphenylmethyl Methacrylate / Asymmetric Anionic Polymerization / Helix / Oligomerization / Protonation / Methylation / (-)-Sparteine / 9-Fluorenyllithium / Stereochemistry /

Conformationally rigid polymers are drawing attention because of their characteristic structures and potential in application for the fields of molecular assembly, chiral recognition, and liquid crystalline formation. ${ }^{1-4} \mathrm{We}$ have been investigating the asymmetric (helixsense-selective) polymerization of bulky methacrylates which give one-handed helical, optically active polymers. A typical example is the polymerization of triphenylmethyl methacrylate (TrMA) with optically active initiators such as the complex of butyllithium ${ }^{5}$ with (-)sparteine $(\mathrm{Sp})$ and the complexes of 9-fluorenyllithium $(\mathrm{FlLi})^{6}$ with $\mathrm{Sp},(+)$ - or (-)2,3-dimethoxy-1,4-bis(dimethylamino)butane, and (+)-1-(2-pyrrolidinylmethyl)pyrrolidine. Recently, we have reported the detailed stereochemical mechanism of the asymmetric polymerization of TrMA. ${ }^{6}$ Although the recent work described the stereochemistry of termination reaction, its scope was limited to protonation using methanol. Here we extend our previous studies to methylation with methyl iodide and protonation with various alcohols in order to obtain information about the effect of the reaction mode in termination (C-C bond formation or protonation) and the stereostructure of the alcohol on the stereochemistry of termination. The termination stereochemistry is interesting because it should reasonably have correlation with the structure and activity of propagating species and therefore it can be a probe into the nature of the propagating species. The examples of studies on the termination stereochemistry in anionic and related polymerizations include the polymerization systems of vinyl- and isopropenylpyridines, ${ }^{7,8}$ phenyl vinyl sulfoxide, ${ }^{9,10}$ 


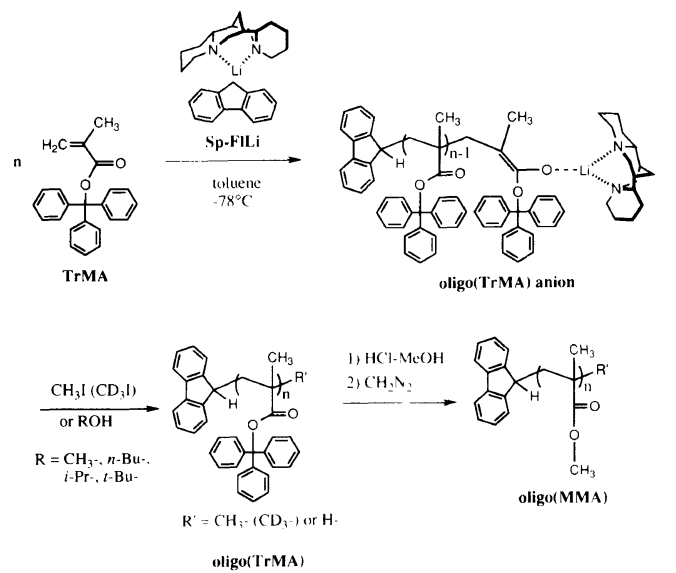

Scheme 1. Asymmetric oligomerization of TrMA, methylation and protonation of oligo(TrMA) anions, and conversion of the resulting oligo(TrMA)s into oligo(MMA)s.

and methyl methacrylate. ${ }^{11-15}$

In the present study, the asymmetric oligomerization of TrMA was carried out with $\mathrm{Sp}-\mathrm{FlLi}$ complex in toluene at $-78^{\circ} \mathrm{C}$. The reaction was terminated by methylation with methyl iodide $\left(\mathrm{CH}_{3} \mathrm{I}\right)$ and methyl iodide- $\mathrm{d}_{3}$ $\left(\mathrm{CD}_{3} \mathrm{I}\right)$ or protonation by methanol, butanol, 2-propanol, and $t$-butanol and the resulting oligomers were converted to oligo(methyl methacrylate) [oligo(MMA)] (Scheme 1). The structure of the oligomers was investigated by means of high performance liquid chromatography (HPLC) separation, ${ }^{1} \mathrm{H}$ NMR spectroscopy, and field desorption (FD) mass spectrometry. On the basis of the obtained results, the stereochemistry of termination is discussed.

\section{EXPERIMENTAL}

\section{Materials}

$\mathrm{CH}_{3} \mathrm{I}$ and $\mathrm{CD}_{3} \mathrm{I}$ (Aldrich) were dried over $\mathrm{CaH}_{2}$ in the dark and distilled under high vacuum immediately before use. Methanol, butanol, 2-propanol, and $t$-butanol were distilled and dried over molecular sieves 4A under $\mathrm{N}_{2}$ atmosphere. Purification of other materials has been described before. ${ }^{6 a}$



Figure 1. Glass ampoule used in the present work.

\section{Oligomerization Procedure and Termination Reaction}

Oligomerization reaction was carried out in the same manner as previously described ${ }^{6 a}$ using a glass ampoule with four side tubes as illustrated in Figure 1. The oligomerization reaction was done in the main tube and the reaction mixture was divided into five aliquots in the side tubes and the main tube after the reaction had completed. The side tubes were sealed and detached from the main tube. The tubes were opened under dry $\mathrm{N}_{2}$ atmosphere and the terminating reagents were added to each aliquot at $-78^{\circ} \mathrm{C}$. The tubes were again sealed and the termination reaction mixtures were warmed to room temperature after standing for $5 \mathrm{~h}$ at $-78^{\circ} \mathrm{C}$. The resulting oligomers were isolated by removing solvent and were converted to oligo(MMA).

\section{Measurements}

${ }^{1} \mathrm{H}$ NMR spectra were taken on a JEOL GX-500 (500 MHz) and a FX-100 (100 MHz) spectrometers. Measurements for MMA-dimer and trimer were done in $\mathrm{CDCl}_{3}$ at $35^{\circ} \mathrm{C}$ and MMA-pentamer in nitrobenzene- $d_{5}$ at $100^{\circ} \mathrm{C}$. Two dimensional NMR spectra of MMA-pentamer were measured under the conditions described before. ${ }^{6 a}, 16$ FD mass spectra were measured on a JEOL DX-HF-303 spectrometer. Chromatographic conditions are the same as described previously. ${ }^{6 a}$ Resolution of MMA-dimer and trimer having two methyl 
groups at the $\omega$-end was done using a column $(25 \times 0.46 \mathrm{~cm}$ (i.d.) $)$ packed with cellulose tris(3,5-dichlorophenylcarbamate)-coated silica gel $^{17}$ with a hexane-2-propanol $(95: 5, \mathrm{v} / \mathrm{v})$ mixture as eluent (flow rate, $0.5 \mathrm{ml} \mathrm{min}^{-1}$ ).

\section{RESULTS AND DISCUSSION}

\section{Methylation Stereochemistry}

The oligomerization reaction of TrMA with $\mathrm{Sp}-\mathrm{FILi}$ complex was carried out at [TrMA]/ [Li] ratios of 2, 3, and 5 and terminated by $\mathrm{CH}_{3} \mathrm{I}$ or $\mathrm{CD}_{3} \mathrm{I}$. In all the reactions, the IR spectra of the reaction mixtures showed no clear sign of unreacted TrMA monomer, indicating that the reactions were quantitative. The GPC curves of oligo(MMA)s derived from the obtained oligo(TrMA)s did not show any clear difference from those of the oligomers terminated by methanol reported in the previous paper. ${ }^{6 a}$ Figure 2 shows the FD mass spectra of the oligo(MMA)s derived from oligo(TrMA)s obtained at [TrMA $] /[\mathrm{Li}]$ ratios of 3 and 5. The spectra showed peaks at $\mathrm{m} / \mathrm{z}$ of $380,480,580,680,780,880$, and 980 which
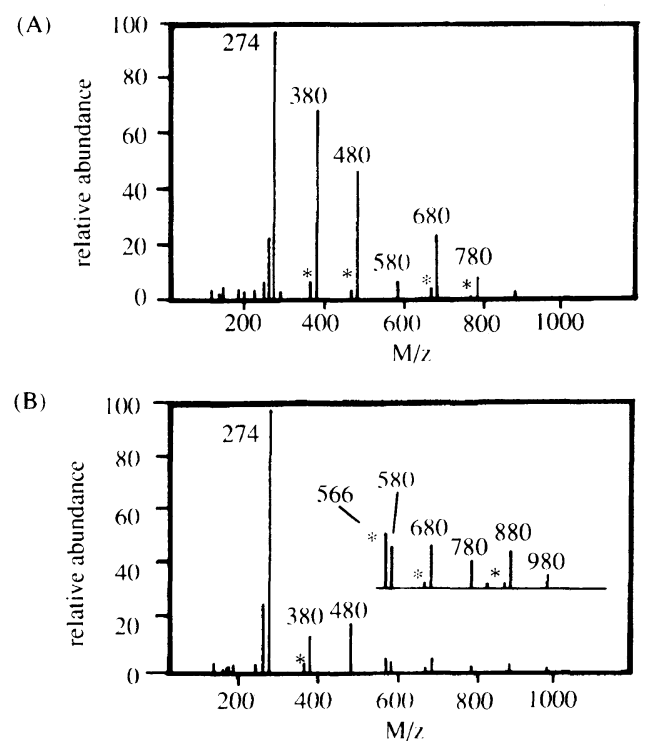

Figure 2. FD mass spectra of oligo(MMA)s derived from oligo(TrMA)s prepared at $[\operatorname{TrMA}] /[\mathrm{Li}]=3(\mathrm{~A})$ and $5(\mathrm{~B})$ with $\mathrm{CH}_{3} \mathrm{I}$ as terminating reagent. correspond to the molecular weight of oligo(MMA)s having a fluorenyl group at the $\alpha$ end and two methyl groups at the $\omega$-end. One of the two methyl groups originates from $\mathrm{CH}_{3} \mathrm{I}$. The large peak at $m / z$ of 274 corresponds to methyl tripheylmethyl ether produced in the process of conversion of oligo(TrMA) to oligo(MMA). The peaks corresponding to the molecular weight of oligomers having a hydrogen at the $\omega$-end, the asterisked peaks at $m / z$ of $165+100 n+1$ in which $n$ represents the degree of polymerization (DP), had much less intensity, indicating that the yield of methylation was high enough to discuss the structure of oligomer anion on the basis of the present results. Exceptionally, the peak at $\mathrm{m} / \mathrm{z}$ of 566 corresponding to the tetramer having a hydrogen at the $\omega$-end had a considerable intensity. The tetramer anion generated at the [TrMA $] /[\mathrm{Li}]$ ratio of 5 appears to be less reactive toward $\mathrm{CH}_{3} \mathrm{I}$ than the other anions. This may be based on some conformational difference between the tetramer anion and the other anions. The series of peaks at $m / z$ values of $350,450,550,-----$, which were seen in our previous work and were assumed to be based on the side products in ester conversion from tripheylmethyl to methyl, ${ }^{6 a}$ are not clearly observed in the spectra.

Figure 3 shows the chromatograms of dia-

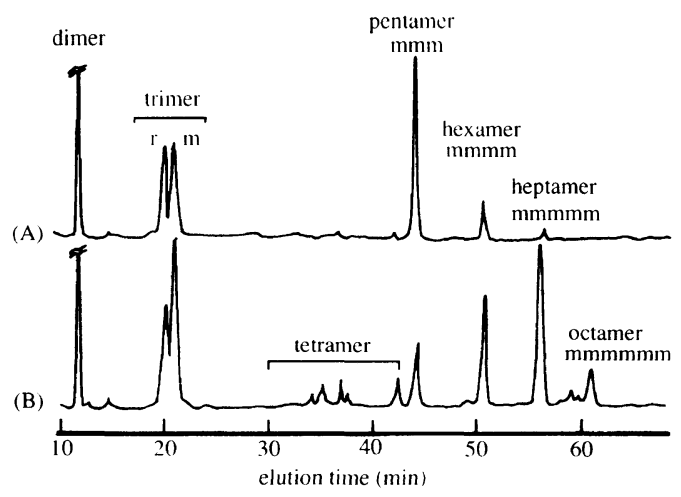

Figure 3. HPLC chromatograms of separation of the oligo(MMA)s derived from oligo(TrMA)s prepared at $[$ TrMA $] /[\mathrm{Li}]=3(\mathrm{~A})$ and $5(\mathrm{~B})$ with $\mathrm{CH}_{3} \mathrm{I}$ as terminating reagent. 
Table I. ${ }^{1} \mathrm{H}$ NMR chemical shift values $(\delta, \mathrm{ppm})$ of MMA-dimer, trimer, and pentamer having a 9-fluorenyl group at the $\alpha$-end and two methyl groups at the $\omega$-end ${ }^{\text {a }}$

\begin{tabular}{|c|c|c|c|c|c|c|c|c|c|c|c|c|}
\hline & \multirow{2}{*}{$\begin{array}{l}\text { Fluorenyl } \\
\text { methine }^{b}\end{array}$} & \multicolumn{2}{|c|}{$\alpha_{1}$} & \multicolumn{2}{|c|}{$\alpha_{2}$} & \multicolumn{2}{|c|}{$\alpha_{3}$} & \multicolumn{2}{|c|}{$\omega_{2}$} & \multicolumn{2}{|c|}{$\omega_{1}$} & \multirow[b]{2}{*}{$\mathrm{OCH}_{3}{ }^{\mathrm{h}}$} \\
\hline & & $\mathrm{CH}_{2}{ }^{\mathrm{c}}$ & $\mathrm{CH}_{3}{ }^{\mathrm{d}}$ & $\mathrm{CH}_{2}{ }^{\mathrm{e}}$ & $\mathrm{CH}_{3}{ }^{\mathrm{d}}$ & $\mathrm{CH}_{2}{ }^{\mathrm{e}}$ & $\mathrm{CH}_{3}{ }^{\mathrm{d}}$ & $\mathrm{CH}_{2}{ }^{\mathrm{e}}$ & $\mathrm{CH}_{3}{ }^{\mathrm{d}}$ & $\mathrm{CH}_{2}{ }^{\mathrm{f}}$ & $\mathrm{CH}_{3}{ }^{\mathrm{g}}$ & \\
\hline Dimer & 3.86 & 2.06 & 1.32 & & & & & & & 2.19 & 1.21 & 3.65 \\
\hline & & 2.43 & & & & & & & & 2.21 & 1.14 & $\begin{array}{l}3.64 \\
3.63\end{array}$ \\
\hline \multicolumn{13}{|l|}{ Trimer } \\
\hline$m$ & 3.84 & $\begin{array}{l}2.06 \\
2.36\end{array}$ & 1.28 & $\begin{array}{l}2.13 \\
2.25\end{array}$ & 1.17 & & & & & $2.07^{i}$ & $\begin{array}{l}1.10 \\
1.05\end{array}$ & $\begin{array}{l}3.65 \\
3.64^{\mathrm{j}}\end{array}$ \\
\hline$r$ & 3.86 & $\begin{array}{l}2.00 \\
2.44\end{array}$ & 1.26 & $\begin{array}{l}1.76 \\
2.33\end{array}$ & 1.16 & & & & & $2.09^{i}$ & $\begin{array}{l}1.16 \\
1.07\end{array}$ & $\begin{array}{l}3.64^{\mathrm{j}} \\
3.57\end{array}$ \\
\hline $\begin{array}{r}\text { Pentamer } \\
\text { mmmm }\end{array}$ & 3.78 & $\begin{array}{l}1.99 \\
2.38\end{array}$ & 1.50 & $\begin{array}{l}1.84 \\
2.48\end{array}$ & 1.36 & $\begin{array}{l}1.75 \\
2.38\end{array}$ & 1.34 & $\begin{array}{l}1.65 \\
2.28\end{array}$ & 1.21 & $1.97^{\mathrm{i}}$ & $\begin{array}{l}1.11 \\
1.07\end{array}$ & $\begin{array}{l}3.66 \\
3.60^{\mathrm{j}} \\
3.58 \\
3.56\end{array}$ \\
\hline
\end{tabular}

${ }^{a}$ The spectra of dimer and trimer were taken in $\mathrm{CDCl}_{3}$ at $35^{\circ} \mathrm{C}(100 \mathrm{MHz})$ and that of pentamer in nitrobenzene- $d_{6}$ at $100^{\circ} \mathrm{C}(500 \mathrm{MHz})$. Aromatic protons of fluorenyl group resonated in the range of 7.2 to $7.7 \mathrm{ppm}$ both in $\mathrm{CDCl}_{3}$ and nitrobenzene- $d_{6}$. Numbering system of monomeric units of the oligo(MMA)s is shown below:

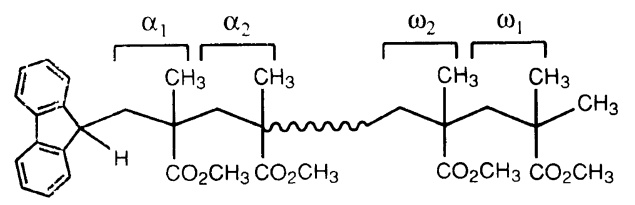

${ }^{\mathrm{b}}$ Triplet. ${ }^{3} J$ coupling constants between the methine proton and the $\alpha_{1}$-methylene protons were in the range of 4 to $5 \mathrm{~Hz} .{ }^{\mathrm{c}}$ Double-quartet. ${ }^{2} \mathrm{~J}$ coupling constants between the geminal protons were $\mathrm{ca} .14 \mathrm{~Hz}$. ${ }^{\mathrm{d}}$ Singlet. ${ }^{\mathrm{c}} \mathrm{AB}$ quartet. ${ }^{2} J$ coupling constants between the geminal protons were in the range of 13 to $15 \mathrm{~Hz}$. ${ }^{\mathrm{f}}$ Singlet or two singlets. ${ }^{\mathrm{g}}$ Two singlets. ${ }^{\mathrm{h}}$ Singlets. The assignments have not been done. ${ }^{\mathrm{i}}$ The peak area corresponds to two protons. ${ }^{\mathrm{j}}$ The peak area corresponds to six protons.

stereomeric separation of oligo(MMA) samples, which were subjected to the mass spectrometry analysis (Figure 2), by HPLC with a silica gel column. The fractions were collected and structural analyses were done using FD mass and ${ }^{1} \mathrm{H}$ NMR. By the HPLC experiment, the trimer was separated in to the two diastereomers, meso $(m)$ and racemo $(r)$ while the dimer showed only one peak which was consistent with the fact that the dimer has no diastereomers. The oligomers with DP of 5 or larger prepared by termination with methanol have been found to consist mainly of the two isotactic oligomers which are different only in the $\omega$-end configuration. ${ }^{6 a}$ The oligomers with DP of 5 or larger in Figure 3 consist almost purely of one peak for each because there is no distinction of the $\omega$-end dyad configuration for the isotactic oligomers.

The chemical shifts of the peaks of ${ }^{1} \mathrm{H}$ NMR spectra of the oligo(MMA)s are shown in Table I. The chemical shift values for the oligomers having two methyl groups at the $\omega$-end are fairly close to those for the oligomers having a hydrogen at the $\omega$-end. ${ }^{6 a}$ The only clear difference was seen in the signals of the nonequivalent methylene protons of $\omega$-end monomeric unit ( $\omega_{1}$-monomeric unit); the methylene protons of the oligomers having two methyl groups showed no splitting. This suggests that the spectroscopic nonequivalency of the $\omega$-end methylene protons is caused 

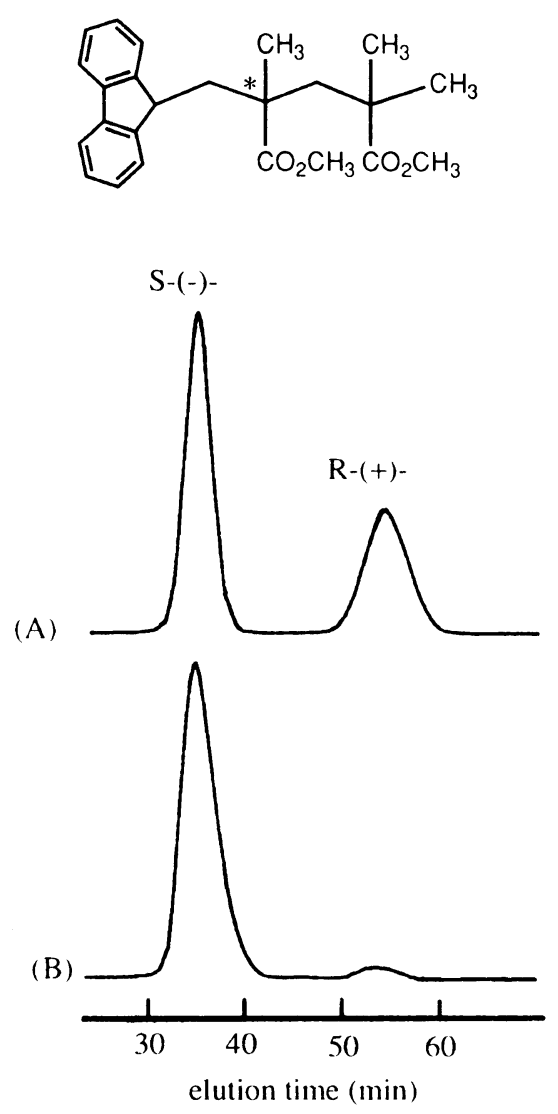

Figure 4. Resolution chromatograms of MMA-dimers having two methyl groups at the $\omega$-end. The dimers were isolated by GPC from the oligo(MMA) mixtures derived from oligo(TrMA)s prepared at $[\mathrm{TrMA}] /[\mathrm{Li}]$ ratios of 2 (A) and 3 (B).

mainly by the chirality of the asymmetric center of $\omega$-end monomeric unit rather than that of the asymmetric center of the penultimate monomeric unit ( $\omega_{2}$-monomeric unit).

The MMA-dimers and -trimers derived from the oligo(TrMA)s obtained at different [TrMA] $/[\mathrm{Li}]$ ratios were resolved by chiral HPLC $^{17}$ (Figures 4 and 5). These oligomers were separated from the crude oligo(MMA) as a mixture of stereoisomers by means of GPC prior to the resolution. The assignments of the peaks were reasonably done by comparing the ratio of peak areas to the ratio of isomers of dimers and trimers obtained from the reac-<smiles>COC[C@@](C)(CC(C)(C)C[C@](C)(CC1c2ccccc2-c2ccccc21)C(=O)OC)C(=O)OC</smiles>

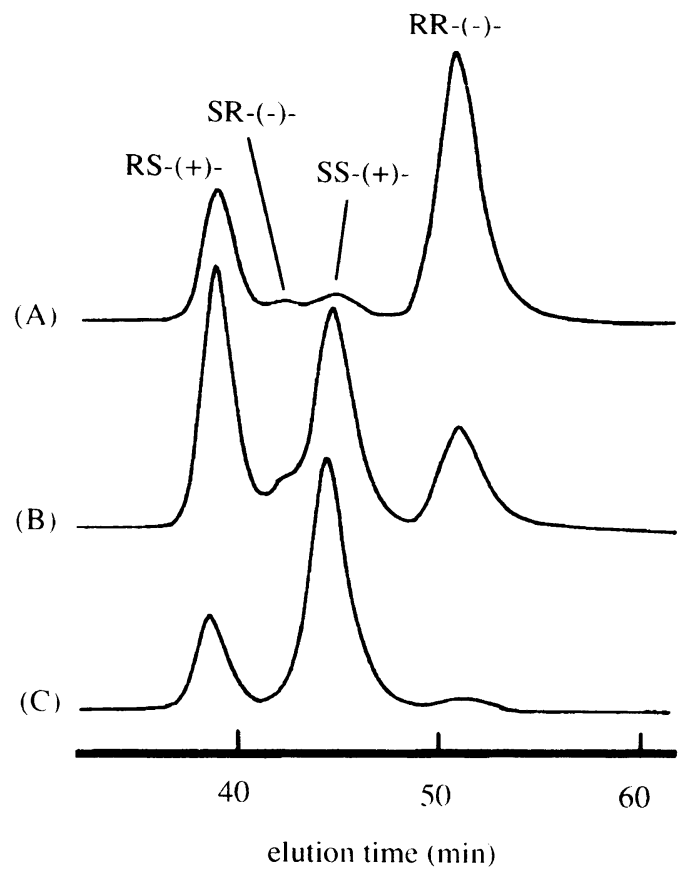

Figure 5. Resolution chromatograms of MMA-trimers having two methyl groups at the $\omega$-end. The trimers were isolated by GPC from the oligo(MMA) mixtures derived from oligo(TrMA)s prepared at [TrMA $] /[\mathrm{Li}]$ ratios of 2 (A) and 3 (B).

tion system with methanol as a terminating reagent. ${ }^{6 a}$ These assignments are based on the assumption that the ratio of $R$-dimer to $S$ dimer and the content of $R R$-, $S S$-, $S R$-, and $R S$-trimers correspond to the isomer content of the dimer and the trimer obtained with methanol as a termination reagent because the termination with $\mathrm{CH}_{3} \mathrm{I}$ should not affect the chirality of the asymmetric center except for that of the $\omega$-end unit.

In order to obtain further information on the stereochemistry of methylation of dimer anion, the termination was also done with $\mathrm{CD}_{3} \mathrm{I}$ at $[\mathrm{TrMA}] /[\mathrm{Li}]$ ratio of 2 and the 


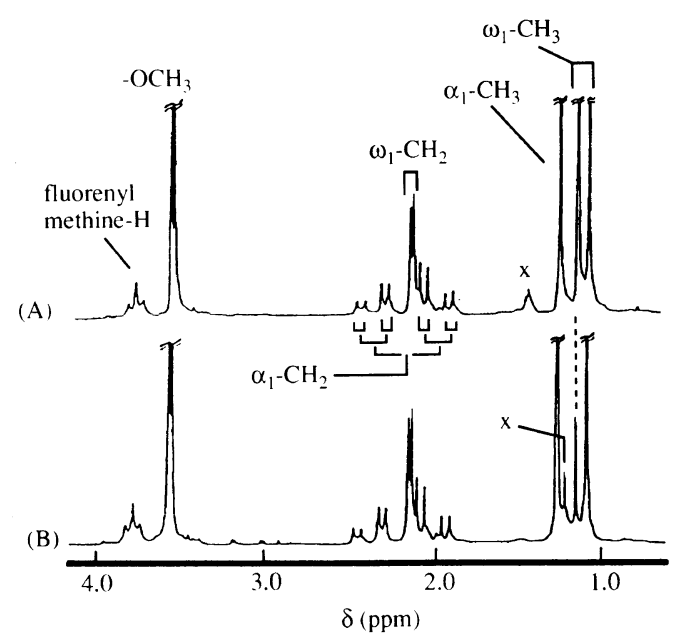

Figure 6. ${ }^{1} \mathrm{H}$ NMR spectra of MMA-dimers having two methyl groups at the $\omega$-end derived from TrMA-dimers prepared with $\mathrm{CH}_{3} \mathrm{I}(\mathrm{A})$ and $\mathrm{CD}_{3} \mathrm{I}(\mathrm{B})$ as terminating reagent.

obtained dimer was converted to MMA-dimer. Figure 6 shows the ${ }^{1} \mathrm{H}$ NMR spectra of MMA-dimers obtained by the termination with $\mathrm{CH}_{3} \mathrm{I}$ and $\mathrm{CD}_{3} \mathrm{I}$. In the spectrum of MMAdimer having a methyl group originating from $\mathrm{CH}_{3} \mathrm{I}$ (Figure 6(A)), three methyl signals of equal intensity were observed. The peak of the lowest magnetic field ( $c a .1 .2 \mathrm{ppm}$ ) was assigned to that of methyl group of the $\alpha_{1}$-monomeric unit since the chemical shift was close to that of the $\alpha_{1}$-methyl group of both $m$ and $r$ MMA-dimers having a hydrogen at the $\omega$-end. The other two peaks are ascribed to the methyl groups at the $\omega$-end: the two methyl groups are diastereotopic on the basis of the chirality of asymmetric center of $\alpha_{1}$-monomeric unit. In contrast to the spectrum (A), in the spectrum (B) of the MMA-dimer having a methyl- $d_{3}$ group originating from $\mathrm{CD}_{3} \mathrm{I}$, one of two $\omega$-end methyl signals is much smaller than the other. This indicates that the methylation of dimer anion by $\mathrm{CD}_{3} \mathrm{I}$ was stereoselective. The intensity ratio of the two peaks, which means the selectivity, was $1 / 4$.

Hogen-Esch and coworkers have reported the methylation stereochemistry of methyl methacrylate $^{13,14}$ and 2-isopropenylpyridine ${ }^{8}$ oligomerizations: they also observed two singlet peaks for two diastereotopic methyls at the $\omega$-end of oligo(MMA) and oligo(2-isopropenylpyridine). They assigned the peak in higher magnetic field, i.e., the one with a smaller chemical shift value in ppm unit, as "pro-m" and the other at "pro-r" for both oligo(MMA) and oligo(2-isopropenylpyridine) on the basis of crystal structure of syndiotactic 2-isopropenylpyridine tetramer and the similarity in the ${ }^{1} \mathrm{H}$ NMR spectra of oligo(2isopropenylpyridine)s and oligo(MMA)s. "Pro- $m$ " methyl at the $\omega$-end has the same position as that of the $\omega$-end hydrogen atom in meso $\omega$-end configuration of the oligomer or polymer obtained from the corresponding anion by protonation.

If this assignment is valid also for our case, our results should mean that the methylation of TrMA-dimer anion occurs predominantly in racemo fashion similarly to the protonation with $\mathrm{MeOH}$ while a TrMA monomer attacks the dimer anion exclusively in meso fashion. This suggests that the reaction mode (C-C bond formation or protonation) has much smaller effect on the termination stereochemistry than the bulkiness and stereostructure of a terminating reagent.

\section{Protonation Stereochemistry}

In order to elucidate the influence of protonating reagents on the stereochemistry of protonation, the oligomerization reactions were carried out at the $[\mathrm{TrMA}] /[\mathrm{Li}]$ ratios of 2, 3, and 5 and terminated with methanol, butanol, 2-propanol, and $t$-butanol. The GPC curves of the oligo(MMA)s derived from the oligo(TrMA)s obtained in the three systems with butanol, 2-propanol, and $t$-butanol were almost identical to that of the oligo(MMA)s obtained from the system with methanol as a terminating reagent. This indicates that the structure of the terminating alcohols does not affect the distribution of oligomers. The content of stereoisomers in each oligomer was determined in the same manner as described 
Table II. The ratio of four isomers of MMA-dimer derived from TrMA-dimer prepared with $\mathrm{Sp}-\mathrm{FlLi}$ at $[\mathrm{TrMA}] /[\mathrm{Li}]$ ratio of 2 and protonated with four alcohols ${ }^{\mathrm{a}}$

\begin{tabular}{|c|c|c|c|c|c|c|}
\hline \multirow{3}{*}{ Alcohol } & \multicolumn{4}{|c|}{ Four isomers $/ \%$} & & \\
\hline & \multicolumn{2}{|c|}{ Meso } & \multicolumn{2}{|c|}{ Racemo } & \multicolumn{2}{|c|}{ e.e. $/ \%^{b}$} \\
\hline & $S S-(+)-$ & $R R-(-)-$ & $R S-(-)-$ & $S R-(-)-$ & $\alpha_{1}$-Chiral center & $\omega_{1}$-Chiral center \\
\hline Methanol & 14 & 3 & 29 & 54 & 36 & -15 \\
\hline Butanol & 16 & 3 & 29 & 52 & 36 & -10 \\
\hline 2-Propanol & 19 & 3 & 29 & 49 & 36 & -4 \\
\hline$t$-Butanol & 29 & 3 & 30 & 38 & 34 & 18 \\
\hline
\end{tabular}

${ }^{a}$ Determined by HPLC resolution with a UV detector $(254 \mathrm{~nm})$. The dimer sample for resolution was isolated from oligo (MMA) mixture by GPC. The structure of dimer is shown below with the numbering system of monomeric units:

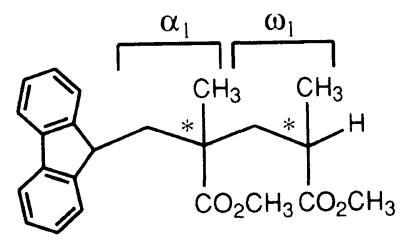

${ }^{\mathrm{b}}$ Excess in $S$ configuration.

previously. ${ }^{6 a}$ In the following text, the stereoisomeric oligomer anions are denoted as $---R^{-}$and $---S-^{-}$with the $\omega$-end configuration neglected since it is not determined until the protonation is completed.

The ratio of four isomers $(S S, R R, R S, S R)$ and e.e. $(\%)$ of the two chiral centers are summarized in Table II. Although the ratio of the four isomers in the system with methanol in the present study is slightly different from that in our previous work, ${ }^{6 a}$ the deviation is considered to be within an experimental error which is probably based on a slight difference in $[\operatorname{TrMA}] /[\mathrm{Li}]$ feed ratio in the oligomerization reaction.

As can be seen from the table, e.e. value for the chiral center of the $\alpha_{1}$-monomeric unit is almost constant regardless of the structure of alcohols, meaning that protonation does not induce racemization of the $\alpha_{1}$-chiral center of dimer anion. Contrastively, e.e. for the chiral center of the $\omega_{1}$-monomeric unit dramatically changed. However, the systematic change was seen only for the content for $S S$ and $S R$ isomers; with the increase in bulkiness of alcohol, the content of $S S$ isomer increased at the expense of $S R$ isomer content. This indicates that bulkiness of the alcohols influences the protonation stereochemistry of only $\mathrm{S}^{-}$- isomeric dimer anion which has been found to be less active in the reaction with TrMA monomer compared to $R^{-}$anion in the early stage of the polymerization. ${ }^{6 a}$ The protonation with a bulkier alcohol tends to result in a larger excess in $S$ configuration of the $\omega$-end.

The effect of alcohol structure on the protonation stereochemistry of timer anion was also investigated for the reaction at [TrMA]/ [Li] ratio of 3 (Table III). The ratio of the eight isomers in the system with methanol is slightly different from the previously reported val$u^{6 a}$ similarly to the case of the dimers. E.e. values for the chiral centers of the $\alpha_{1}$ - and $\alpha_{2}$-monomeric units were almost constant, proving again that the protonation by alcohols does not affect the configuration of the chiral center except for that of the $\omega_{1}$-monomeric unit. A clear change in the content of isomers was observed for the pair of $S S S$ and $S S R$ 


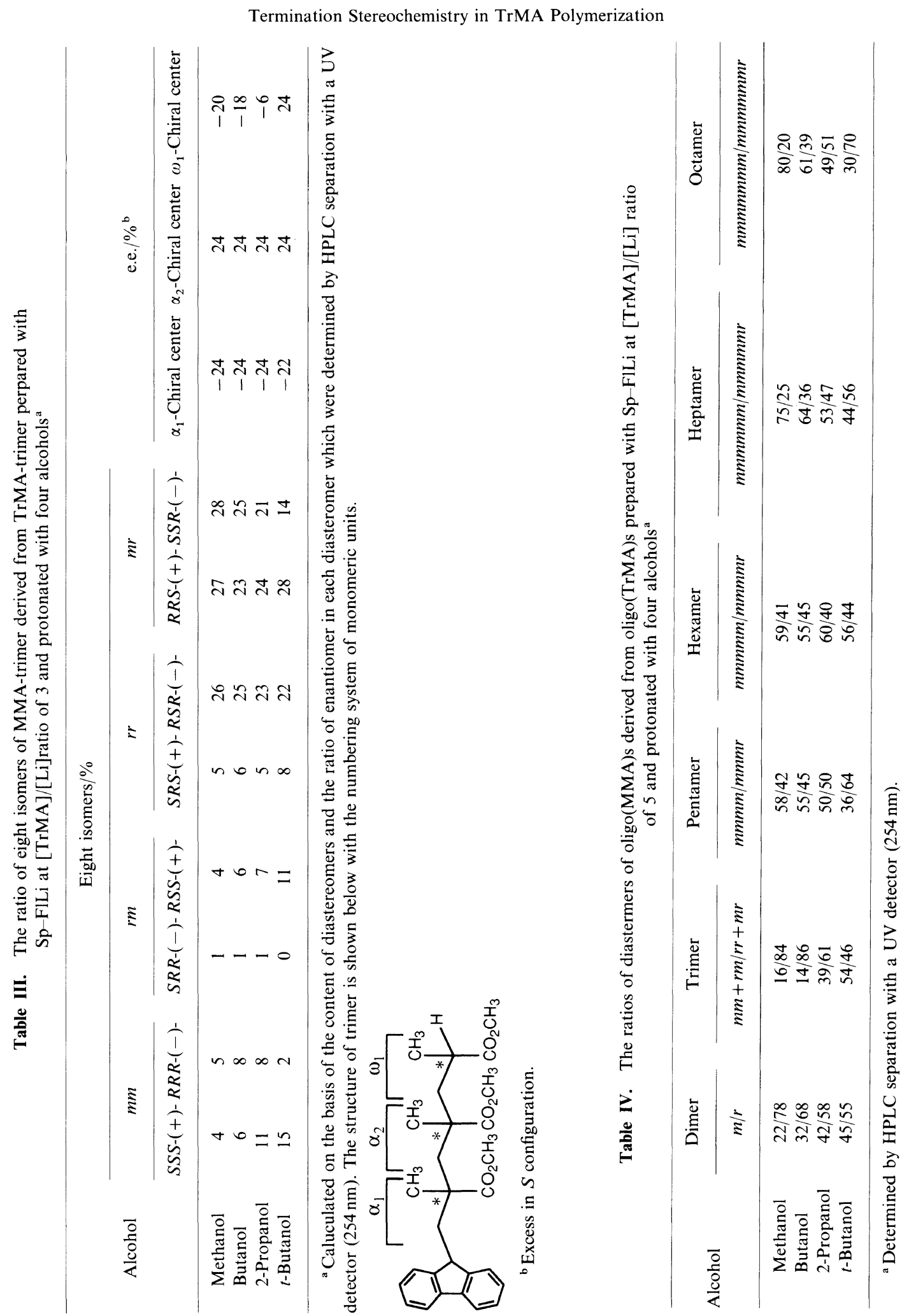

Polym. J., Vol. 27, No. 9, 1995 
produced from $S S$ - $^{-}$anion and for that of $R S S$ and $R S R$ produced from $R S-^{-}$trimer anion. The $S S^{-}{ }^{-}$and $R S-^{-}$anions have been found to be the less active ones toward TrMA monomer. ${ }^{6}$ Similarly to the case of dimer anion, protonation producing $S$ configuration tended to occur when a bulky alcohol was used. These results indicate that the termination stereochemistry of the less active dimer and trimer anions in the propagation is more sensitive to the structure of alcohols in the protonation than that of the anions predominantly reacting with TrMA monomer. This is probably because the less reactive anions are sterically more hindered.

The protonation stereochemistry of the isotactic oligomer anions with DP of 5-8 was also studied by examining the ratio of the isomer having $m \omega$-end configuration to that having $r$ configuration. In the present reaction system, the isotactic isomers are the predominant diastereomers for the oligomers with DP of 5-8. ${ }^{6}$ Also, these oligomers have been found to consist of almost purely one antipode of enantiomers whose chiral centers of the main chain have $R$ configuration except for the hexamer $(\mathrm{DP}=6) \mathrm{mmmr}$; hexamer mmmr consisted of $54 \%$ of $R R R R R S$ isomer and $46 \%$ of $S S S S S R$ isomer in the system with methanol as a terminating reagent. ${ }^{6 a}$ Therefore, it is safe to assume that the change in diastereomer content of pentamer, heptamer, and octamer respectively reflects the protonation chemistry of $R R R R_{-}^{-}, R R R R R^{-}, R R R R R R^{-}$anions. The ratio of the diastereomers of dimer to octamer are summarized in Table IV. As well as at $[\mathrm{TrMA}] /[\mathrm{Li}]$ ratio of 2 (Table II), the contents of $m$ dimer and $r m+m m$ trimer increased with the increasing bulkiness of the alcohols, at [TrMA $] /[\mathrm{Li}]$ ratio of 5 . In contrast to this, as for the pentamer, heptamer, and octamer, the fraction of isotactic oligomers with $r \omega$-end configuration increased with increasing bulkiness of the alcohols. This tendency is more clear for octamer than for pentamer and heptamer. These results indicate that the protonation of isotactic pentamer, heptamer, and octamer anions having $R R R$--configuration tends to be in $r$ fashion producing $S$ configuration at the $\omega$-end when a bulky alcohol was used. On the other hand, the ratio of $m m m m m$ and $m m m m r$ in the hexamer did not show clear tendency of increase or decrease with changing bulkiness of alcohols. This suggests two possibilities: 1) the termination stereochemistry of hexamer anion is not sensitive to the stereostructure of protonating reagent, or 2) the change of protonation mode ( $m$ or $r$ ) of $R R R R R-^{-}$hexamer anion is opposite to that of $S S S S S$ - - hexamer anion resulting in no obvious change in the ratio of mmmmm to $\mathrm{mmmmr}$. Assuming that the latter is more plausible, the termination stereochemistry of SSSSS- ${ }^{-}$anion must be more sensitive to the stereostructure of alcohols than that of $R R R R R^{-}$anion because the amount of $R R R R R^{-}$anion is higher than that of $S S S S S-^{-}$anion: the ratio of $R R R R R^{-}$to $S S S S S$ - $^{-}$anions is calculated to be 83 to 17 using the ratio of $m m m m m$ to $m m m m m r$ $(67: 33)$ and the reported content of enantiomers (RRRRRR:SSSSSS $=97: 3, R R R$ $R R S: S S S S S R=54: 46)$ at the same [TrMA]/ [Li] ratio. ${ }^{6 a}$ Consequently, $S S S S S-^{-}$anion which has been found to be less reactive in propagation may be more sterically crowded around the $\omega$-end anion similarly to the less reactive dimer and trimer anions mentioned above.

\section{CONCLUSION}

Methylation and protonation stereochemistries of oligo(TrMA) anion were investigated. The study on methylation of dimer anion with $\mathrm{CH}_{3} \mathrm{I}$ and $\mathrm{CD}_{3} \mathrm{I}$ showed that methylation occurs stereoselectively. Protonation experiment with various alcohols revealed that 1) bulkiness (stereostructure) of alcohols affects the termination stereochemistry and 2) the effect of alcohols was more obvious for the anions which are less active toward TrMA 
monomer. The latter observation suggests that the less reactive anions are probably more sterically hindered.

\section{REFERENCES}

1. Y. Okamoto and T. Nakano, Chem. Rev., 94, 349 (1994).

2. G. Wulff, Angew. Chem., Int. Ed. Engl., 28, 21 (1989).

3. Y. Okamoto and E. Yashima, Prog. Polym. Sci., 15, 263 (1990).

4. Y. Okamoto, CHEMTECH, 144 (1987).

5. Y. Okamoto, K. Suzuki, K. Ohta, K. Hatada, and H. Yuki, J. Am. Chem. Soc., 101, 4763 (1979).

6. (a) T. Nakano, Y. Okamoto, and K. Hatada, J. Am. Chem. Soc., 114, 1318 (1992).

(b) Y. Okamoto, E. Yashima, T. Nakano, and K. Hatada, Chem. Lett., 759, (1987).

7. A. H. Soum and T. E. Hogen-Esch, Macromolecules, 18, 690 (1985).
8. T. E. Hogen-Esch, R. A. Smith, D. Ades, and M. Fontanille, J. Polym. Sci., Polym. Lett. Ed., 19, 309 (1981).

9. M. A. Buse and T. E. Hogen-Esch, Macromolecules, 20, 1509 (1987).

10. M. A. Buse and T. E. Hogen-Esch, J. Am. Chem. Soc., 107, 4509 (1985).

11. K. G. Banerja and T. E. Hogen-Esch, Macromolecules, 27, 2187 (1994).

12. J. L. Baumgarten, A. H. E. Müller, and T. E. Hogen-Esch, Macromolecules, 24, 353 (1991).

13. R. Volpe, T. E. Hogen-Esch, F. Gores, and A. H. E. Müller, Macromolecules, 25, 3553 (1992).

14. R. Volpe and T. E. Hogen-Esch, Macromolecules, 23, 4196 (1990).

15. K. Ute, T. Asada, N. Miyatake, and K. Hatada, Makromol. Chem., Makromol. Symp., 67, 147 (1993).

16. K. Hatada, K. Ute, K. Tanaka, M. Imanari, and N. Fujii, Polym. J., 19, 425 (1987).

17. Y. Okamoto and Y. Kaida, J. Chromatogr. A, 666, 403 (1994); Y. Okamoto, M. Kawashima, and K. Hatada, J. Chromatogr., 363, 173 (1986). 